\title{
BeppoSAX observation of the rich cluster of galaxies Abell 85
}

\author{
G. B. Lima Neto ${ }^{1,2}$, V. Pislar ${ }^{2,3}$, and J. Bagchi ${ }^{4}$ \\ 1 Instituto Astronômico e Geofísico/USP, Av. Miguel Stefano 4200, 04301-904 São Paulo/S.P., Brazil \\ 2 Institut d'Astrophysique de Paris, CNRS, 98bis Bd. Arago, 75014 Paris, France \\ 3 Université du Havre, 25 rue Philippe Lebon, 76600 Le Havre, France \\ 4 Inter-University Centre for Astronomy and Astrophysics (IUCAA), Post Bag 4, Ganeshkhind, Pune 411007, \\ India
}

Received 23 June 2000 / Accepted 15 December 2000

\begin{abstract}
We report the observation of the Intra-Cluster Medium (ICM) of Abell 85 by the X-ray satellite BeppoSAX. We have analysed both the spectrum obtained in the central 8 arcmin circular region centred on the Very Steep Spectrum Radio Source (VSSRS) and the spectra from a number of sub-regions. Analysis of the spectra allowed us to independently obtain new estimates of the temperature, metallicity and line-of-sight hydrogen density column, both globally $\left(T=6.6 \pm 0.3 \mathrm{keV}, Z=0.38 \pm 0.06 Z_{\odot}\right.$ and $\left.N_{\mathrm{H}}=5.5_{-0.7}^{+0.9} 10^{20} \mathrm{~cm}^{-2}\right)$ and locally. These measures are in good agreement with previous measures based on ROSAT and ASCA data. In the region of the VSRSS, we attempted to discriminate between thermal and non-thermal X-ray emission. Although we could not do this unambiguously, we have nonetheless estimated the extended magnetic field using the radio spectrum available for this region. We obtain a lower limit intensity of $0.9 \mu \mathrm{G}$, consistent with our previous estimate. We also derive $\alpha$-elements/iron abundance ratios that turn out to be higher than 1 . Such a result tends to support the burst model for elliptical galaxies, where a strong galactic wind develops early in the galaxy history and type II supernovae (SN) may be the major contributors to the enrichment of the ICM. A two-temperature ICM model was fitted in the central region, yielding a main component with roughly the mean cluster temperature and a cooler component with temperature less than $0.1 \mathrm{keV}$.
\end{abstract}

Key words. galaxies: clusters: Abell 85 - galaxies: magnetic fields - X-rays: galaxies - galaxies: abundances radio continuum: galaxies

\section{Introduction}

The hot $\left(T \approx 10^{8} \mathrm{~K}\right)$ and tenuous (central density, $n_{0} \approx 10^{-3} \mathrm{~cm}^{-3}$ ) X-ray emitting gas found in rich clusters of galaxies is an excellent tool to probe the cluster dynamics, morphology, and history. The intra-cluster gas accounts for $\sim 10-15 \%$ of the total cluster mass and thanks to its short relaxation time scale, it can track the cluster global gravitational potential. The main emission mechanism of the gas is the thermal bremsstrahlung which is proportional to $\sim n^{2} T^{1 / 2}$ ( $n$ is the numerical density of electrons). Other than density and temperature, the observed thermal bremsstrahlung emission may give estimations of the gas metallicity and of the line-of-sight column density of hydrogen. The abundance of metals in the intracluster gas is a sign of early enrichment by material processed in stars. The determination of precise abundances of iron and $\alpha$-elements (nuclei formed by the fusion of $\alpha$ particles in massive stars) can provide strong constraints

Send offprint requests to: G. B. Lima Neto, e-mail: gastao@iagusp.usp.br for the early evolution of galaxies and the galaxy-cluster medium interaction.

In the last few years, the cluster Abell 85 (richness class $1, \mathrm{cD}$ type, optical redshift 0.056 ) has been extensively studied (cf. Pislar et al. 1997; Lima Neto et al. 1997; Bagchi et al. 1998). Using data from the ROSAT satellite detectors PSPC (imagery and spectroscopy) and HRI (high resolution imagery), it has been possible to draw substantial new conclusions about this cluster. Through the use of wavelet techniques, Lima Neto et al. (1997) were able to detect a central excess in the X-ray emission which was identified with a cooling-flow of about 50-150 $M_{\odot} /$ yr (based on a multi-phase model of the gas). Moreover, they have confirmed the presence of 3 small Xray features around the centre (cf. Prestwich et al. 1995), which may be a sign of inhomogeneities in the central cooling-flow. The second most important X-ray feature in Abell 85 is a blob to the south of the main structure (called the "South Blob" hereafter). This blob is definitely a substructure in the X-ray map and coincides with a small group of galaxies (containing the second-brightest cluster 
member). However, these galaxies do not seem to form a gravitationally bound group and may be part of a chain of galaxies extending to the neighbouring cluster Abell 87 (Durret et al. 1998). The radio maps of the South Blob also show intense, very steep spectrum radio emission at meter wavelengths (the VLA map, see below and also Joshi et al. 1986; Bagchi et al. 1998). Most of this emission is diffuse in nature and extends over $4 \operatorname{arcmin}(\sim 400 \mathrm{kpc})$ but is not associated with any particular galaxy. This, and another similar diffuse radio source $\sim 1 \mathrm{Mpc}$ to the northwest (VSSRS 0038-096), are the principal unexplained radio features in Abell 85.

The radio observations of diffuse synchrotron radiation strongly suggest the presence of a large-scale magnetic field, $B$, and relativistic electrons in clusters of galaxies. These electrons interact not only with magnetic fields but also with the $3 \mathrm{~K}$ cosmic microwave background radiation $(\mathrm{CMB})$. The scatter of the electrons and the CMB through the inverse Compton effect (IC/3K) will produce X-ray photons (e.g. Feenberg \& Primakoff 1948; Rephaeli \& Gruber 1988). The best place to look for a co-spatial $\mathrm{IC} / 3 \mathrm{~K}$ and synchrotron radiation is on a very steep spectrum radio source (VSSRS). These sources, possibly the remnants of former radio galaxies but presently not identifiable with galaxies, are commonly known as cluster "radio-relics" and "radio-haloes" (cf. Feretti \& Giovannini 1996; Kronberg 1994 for reviews). They owe their steep radio spectra to radiative energy losses but are prevented from rapid fading due to expansion by the thermal pressure of the surrounding intra-cluster gas (Baldwin \& Scott 1973). These VSSRS are also possibly the tracers of large scale shock waves that form at the intersection of filaments and sheets of galaxies due to gravity driven supersonic flows of extragalactic matter (Enßlin et al. 1998; Miniati et al. 2000; Bagchi et al. 2001).

We have studied the X-ray excess at the location of the extended VSSRS 0038-096 in the western part of Abell 85 using ROSAT PSPC data and the low frequency radio data (Bagchi et al. 1998). Even though ROSAT has a limited spectroscopic range [0.5-2.4] keV, we were able to derive a value for the magnetic field, $B=1.0 \pm 0.1 \mu \mathrm{G}$. We based our estimate on the assumption that the co-spatial radio and X-ray emission are nonthermal synchrotron and IC/3K radiation, respectively, from a common population of relativistic charges.

In this paper, we present new results based on observation of Abell 85 by the X-ray satellite BeppoSAX. We use the spatially resolved spectroscopy capabilities of the MECS and LECS detectors to determine independently the temperature, metallicity and line-of-sight hydrogen density column. Furthermore, we try to separate the thermal from the non-thermal X-ray emission in the regions of the VSSRS and the South Blob, and we give lower limit estimates of the extended magnetic field. In the central region, we determine the properties of the cooling-flow, showing that the X-ray emission in that region is compatible with a metal-rich, 2-temperature plasma. Moreover, when the signal-to-noise ratio is high enough, we estimate the individual abundances of iron, nickel and $\alpha$-elements. Unless otherwise stated, we assume $H_{0}=50 \mathrm{~km} \mathrm{~s}^{-1} \mathrm{Mpc}^{-1}$ and $q_{0}=0.5$ (i.e., 1 arcmin corresponds to $\left.97 h_{50}^{-1} \mathrm{kpc}\right)$.

\section{The data}

Abell 85 was observed in July 1998 by the BeppoSAX satellite (Boella et al. 1997a) with two of the narrow field instruments: the low-energy concentrator spectrometer (LECS, sensitive in the [0.1-10.0] keV range; Parmar et al. 1997) and two units of the medium-energy concentrator spectrometer (MECS 2 and 3, sensitive in the [1.310.5] keV range; Boella et al. 1997b).

The observation was not pointed at the centre of the cluster, at the position of the central cD galaxy, but at the position of the VSSRS 0038-096. The net exposure times were 92708 and $40810 \mathrm{~s}$ for the MECS and LECS, respectively.

\subsection{Data reduction}

The data have been pre-processed (linearized and cleaned) using the SAXDAS Rev. 1.1 package in the FTOOLS 3.5 environment at the BeppoSAX Science Data Centre (SDC) and then retrieved through their archive.

We have used the merged data from both MECS units; they were merged using the SAXDAS "meevelin" program by the SDC (see Fiore et al. 1999).

In order to avoid severe vignetting effects, we have extracted data for scientific analysis only inside a circle of 8 arcmin around the pointing axis of the LECS and MECS instruments (Cusumano \& Mineo 1998).

The background spectra have been extracted from sky fields devoid of detectable sources and using the same regions on the detectors as the ones used for extracting spectra of Abell 85 (see below for the definition of these regions).

\subsection{Data analysis}

The total field of view of the MECS units is $0.5^{\circ}$, but they are held by a beryllium support that blocks photons with $E<5 \mathrm{keV}$; this effect is not taken into account in the effective area files (Fiore, private communication). Therefore, we restricted our analysis to the area inside the support, i.e., up to 8 arcmin from the detector axis.

Both instruments, LECS and MECS, have moderate spatial resolution, $3.7^{\prime}$ at $0.28 \mathrm{keV}$ and $1.2^{\prime}$ at $6.4 \mathrm{keV}$ (FWHM) respectively (Fiore et al. 1999). Hence, we have defined sub-regions in the field of view in order to perform separate spectral analyses. The X-ray data in these regions were extracted with XSELECT 1.4. Table 1 summarizes the characteristics of the regions we have used.

Figure 1 shows the regions we selected superimposed on the X-ray map derived from the MECS23 data. This figure also shows the $90 \mathrm{~cm}$ radio continuum VLA and ROSAT PSPC maps (isocontour lines). For the radio data, 


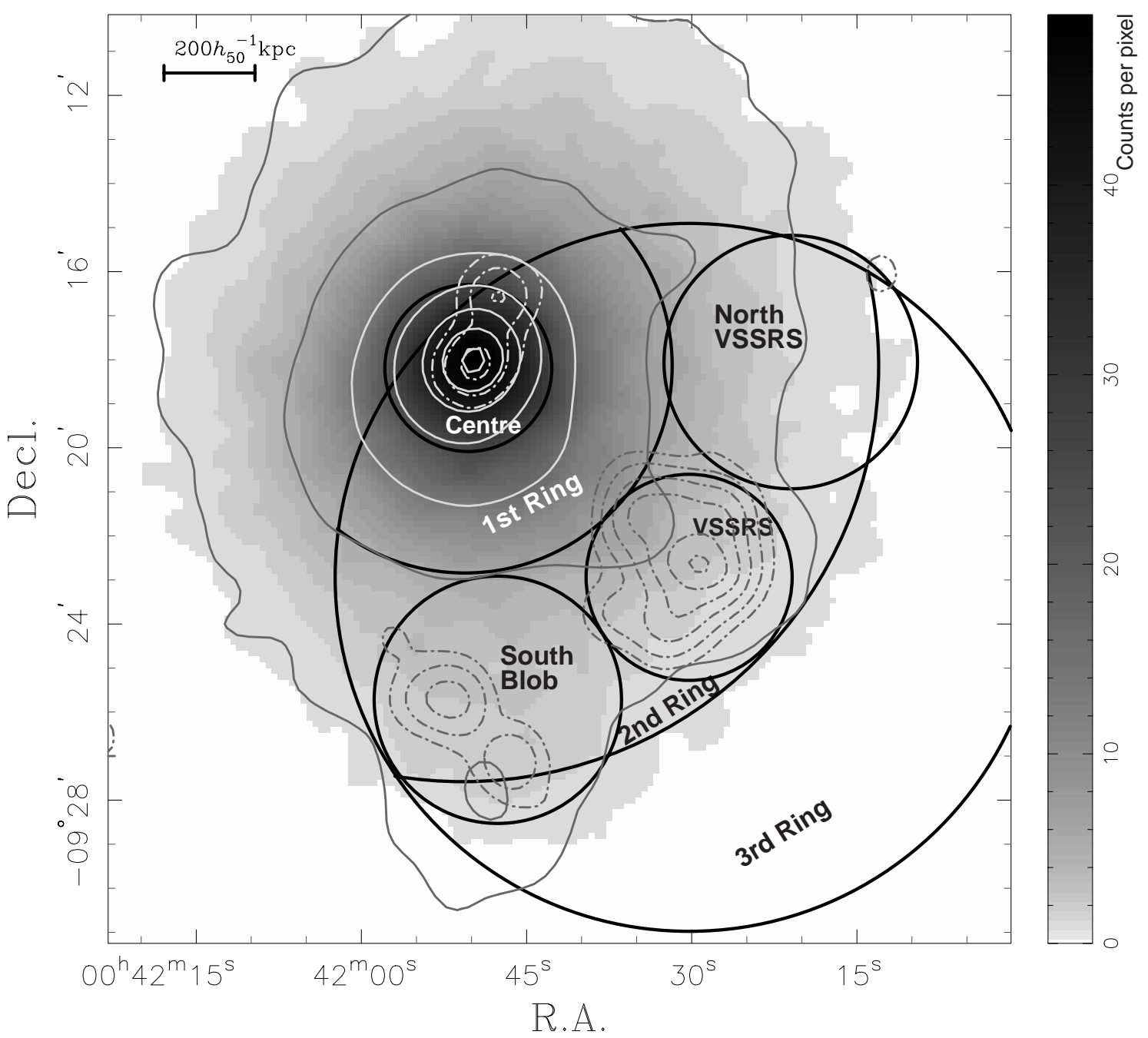

Fig. 1. The regions used for the analysis of MECS and LECS data of Abell 85 are the thick black circles and rings. In grey-scale is the MECS23 image. The continuous thin lines (in white in the centre for the sake of visibility) are contours of the PSPC data smoothed with a $\sigma=0.45$ arcmin gaussian. The dot-dashed thin lines are contours from the VLA radio continuum map at $90 \mathrm{~cm} \lambda$ (see text)

we used the VLA C-configuration, archival interferometric data (programme ID AR279). The data were obtained in July 1993 with a total of $30 \mathrm{~min}$ of integration on Abell 85 at the frequency of $333 \mathrm{MHz}$ and bandwidth of 3.1 MHz. For reduction, the NRAO "AIPS" software was employed. Following the usual procedure of editing and amplitude and phase calibration, the calibrated data was Fourier transformed and the $3 \times 3$ degree image was deconvoluted with the "CLEAN" algorithm. The final image was convoluted with a circular restoring beam of 60 arcsec (FWHM) gaussian profile. Noteworthy is the strong radio emission from the region of VSSRS, the South Blob and the cD galaxy at the cluster centre. The PSPC X-ray map is fully described elsewhere (Pislar et al. 1997).

We have extracted the spectra for each region and used simultaneously both the LECS and MECS (units 2 and 3) data. Following the "Cookbook" (Fiore et al. 1999), we have used only the data in the interval [0.12-4.0] $\mathrm{keV}$ for the LECS and [1.65-10.5] keV for the MECS.
For each region we have used a single temperature plasma, the absorbed Raymond-Smith (Raymond \& Smith 1977) and the MEKAL (Kaastra \& Mewe 1993; Liedahl et al. 1995) models. For some regions (centre, South Blob and VSSRS) we also modelled the intracluster medium with a combination of two models: either two MEKAL or a MEKAL plus a power-law, to take into account non-thermal emission. The absorption is due to the cold gas in the line-of-sight, mainly hydrogen and helium. We have used the photoelectric absorption cross-sections given by Balucinska-Church \& McCammon (1992).

When the counts were high enough, we also tried the VMEKAL model, that is, with variable individual abundances rates for the elements that contribute to the X-ray flux in the [0.1-10.0] keV band. Unfortunately, most of the metals do not produce strong enough lines for unambiguous detection with BeppoSAX. Therefore we fixed the abundance values of $\mathrm{He}, \mathrm{C}, \mathrm{N}, \mathrm{O}, \mathrm{Ne}, \mathrm{Na}, \mathrm{Mg}$, and $\mathrm{Al}$ to $0.3 Z_{\odot}$. 
Table 1. Regions used in the spatial/spectral analysis of Abell 85. The fluxes and luminosities are estimated using an absorbed MEKAL model. Errors are $3 \sigma$

\begin{tabular}{|c|c|c|c|c|}
\hline Name & $\begin{array}{l}\text { Count rate } \\
\text { (counts/s) }\end{array}$ & $\begin{array}{r}\text { Flux } \\
{[2.0-10.0] \mathrm{keV}} \\
\end{array}$ & $\begin{array}{r}L_{\mathrm{X}}^{\dagger} \\
{[2.0-10.0] \mathrm{keV}} \\
\end{array}$ & Localisation \\
\hline Centre & $0.259 \pm 0.002$ & $4.37 \pm 0.31$ & $6.13 \pm 0.41$ & Circle at $0^{\mathrm{h}} 41^{\mathrm{m}} 50^{\mathrm{s}} 27,-9^{\circ} 18^{\prime} 12.0^{\prime \prime}$, radius $1.9^{\prime}$ \\
\hline South Blob & $0.091 \pm 0.001$ & $1.53 \pm 0.21$ & $2.13 \pm 0.29$ & Circle at $0^{\mathrm{h}} 41^{\mathrm{m}} 44^{\mathrm{s}} 33,-9^{\circ} 23^{\prime} 52.0^{\prime \prime}$, radius $3.2^{\prime}$. \\
\hline VSSRS & $0.0331 \pm 0.0006$ & $0.55 \pm 0.09$ & $0.77 \pm 0.12$ & Circle at $0^{\mathrm{h}} 41^{\mathrm{m}} 30.27,-9^{\circ} 22^{\prime} 56.0^{\prime \prime}$, radius $2.3^{\prime}$ \\
\hline North VSSRS & $0.0362 \pm 0.0007$ & $0.61 \pm 0.11$ & $0.85 \pm 0.15$ & Circle at $0^{\mathrm{h}} 41^{\mathrm{m}} 20^{\mathrm{s}} 95,-9^{\circ} 18^{\prime} 04.0^{\prime \prime}$, radius $2.9^{\prime}$. \\
\hline 1st Ring & $0.220 \pm 0.002$ & $3.70 \pm 0.27$ & $5.18 \pm 0.36$ & $\begin{array}{l}\text { Ring between } 2.0^{\prime} \text { and } 4.7^{\prime} \text {, centred at } \\
0^{\mathrm{h}} 41^{\mathrm{m}} 50.27,-9^{\circ} 18^{\prime} 12.0^{\prime \prime} . \text { The region outside } 8^{\prime} \\
\text { from the MECS axis is excluded. }\end{array}$ \\
\hline 2nd Ring & $0.111 \pm 0.001$ & $1.86 \pm 0.19$ & $2.60 \pm 0.25$ & $\begin{array}{l}\text { Ring between } 4.7^{\prime} \text { and } 9.4^{\prime} \text {, centred at } \\
0^{\mathrm{h}} 41^{\mathrm{m}} 50.27,-9^{\circ} 18^{\prime} 12.0^{\prime \prime} . \text { The region outside } 8^{\prime} \\
\text { from the MECS axis is excluded. }\end{array}$ \\
\hline 3rd Ring & $0.01176 \pm 0.0005$ & $1.98 \pm 0.21$ & $0.27 \pm 0.03$ & $\begin{array}{l}\text { Region outside the circle centred at } 0^{\mathrm{h}} 41^{\mathrm{m}} 50.27 \text {, } \\
-9^{\circ} 18^{\prime} 12.0^{\prime \prime} \text { and radius } 9.4^{\prime} \text {, excluding the } \\
\text { region outside } 8^{\prime} \text { from the MECS axis. }\end{array}$ \\
\hline All Field & $0.587 \pm 0.003$ & $9.95 \pm 0.45$ & $13.94 \pm 0.61$ & Circle at $0^{\mathrm{h}} 41^{\mathrm{m}} 30^{\mathrm{s}} 27,-9^{\circ} 22^{\prime} 56.0^{\prime \prime}$ radius $8.0^{\prime}$. \\
\hline
\end{tabular}

* Flux is in units of $10^{-11} \mathrm{erg} \mathrm{s}^{-1} \mathrm{~cm}^{-2}$.

$\dagger$ Luminosity is in units of $10^{44} \mathrm{erg} \mathrm{s}^{-1}$.

The spectral fits were done using XSPEC v10.0. The BeppoSAX narrow-field instruments have channels of equal energy width, but a spectral resolution that scales roughly with the square root of the energy (Boella 1997a). Therefore, if a spectrum must be rebinned (in order to increase the counts per bin), one should first group the bins taking into account the energy resolution nonlinearity rather than simply grouping the channels with some ad hoc prescription. We used the appropriate rebinning template files made available by the SDC (Fiore et al. 1999).

However, even with the energy-dependent rebinning, there are still bins with low counts. We have then used the "recipe" given by Churazov et al. (1996), based on the smoothed rebinned spectrum (available in XSPEC) for computing the statistical weights. With this procedure, one can still use least-square minimisation fit and $\chi^{2}$ statistics for unbiased parameter and error estimation.

\section{Results}

\subsection{All field}

Within the unobstructed field-of-view of a circle of 8 arcmin centred at the MECS axis we have our greatest signal-to-noise ratio and in this region we can obtain some well-constrained mean quantities for Abell 85. Using a MEKAL model, we find a temperature of $6.6 \pm 0.3 \mathrm{keV}$, a hydrogen column density $N_{\mathrm{H}}=5.5_{-0.7}^{+0.9} 10^{20} \mathrm{~cm}^{-2}$ and a metallicity of $0.38 \pm 0.06 Z_{\odot}$ (cf. Table 2). We obtain a significantly higher temperature than that obtained with the ROSAT PSPC $(4 \pm 1 \mathrm{keV}$, Pislar et al. 1997), but essentially the same as the one obtained with ASCA ( $6.1 \pm 0.2 \mathrm{keV}$, but notice that this is the mean temperature within $\sim 15$ arcmin; Markevitch et al. 1998). The column density is higher than the Galactic value deduced from HI data in the field of view (for example $3.0810^{20} \mathrm{~cm}^{-2}$ at the position of the VSSRS and $3.5810^{20} \mathrm{~cm}^{-2}$ at the position of the cD, Dickey \& Lockman 1990). This suggests that there is an amount of $\mathrm{HI}$ contained within the cluster itself.

The metallicity is well-constrained and has the value generally found in this type of cluster (e.g. Fukazawa et al. 1998). We have further fitted the data with a VMEKAL model to obtain abundances of the individual metal elements. The reduced $\chi^{2}\left(\chi^{2}\right.$ divided by the number of degrees of freedom) is slightly higher for the VMEKAL compared to the MEKAL model, but the temperature and hydrogen column density are the same and we detect the presence of some metals (cf. Table 2). Only the abundances of $\mathrm{Ni}$ and $\mathrm{Fe}$ are not compatible with zero at $1 \sigma$ level and, except for these two elements, we can only give upper limits for the abundance.

The total X-ray luminosity in the $[0.1-2.4] \mathrm{keV}$ band is $(8.49 \pm 0.37) 10^{44} h_{50}^{-2} \mathrm{erg} \mathrm{s}^{-1}$ inside a radius of $770 h_{50}^{-1} \mathrm{kpc}$ (8 arcmin) centred at the VSSRS position.

\subsection{Centre}

We take a region of $1.9 \operatorname{arcmin}\left(185 h_{50}^{-1} \mathrm{kpc}\right)$ at the position of the $\mathrm{cD}$ galaxy located at the cluster centre. A fit with a single temperature model shows a significant increase in the hydrogen column density and a decrease in temperature relative to the values obtained when we 
Table 2. Spectral fitting results for single component models. Errors are $3 \sigma$ except when explicitly stated. The abundances are given in Solar units using values from Anders \& Grevesse (1989). All metals, other than those in the table, are fixed at $Z=0.3 Z_{\odot}$

\begin{tabular}{|c|c|c|c|c|c|c|c|c|c|c|c|}
\hline Region & Model & $\begin{array}{c}N_{\mathrm{H}} \\
\left(10^{20} \mathrm{~cm}^{-2}\right)\end{array}$ & $\begin{array}{c}k T \\
(\mathrm{keV})\end{array}$ & $Z$ & $Z_{\mathrm{Si}}$ & $Z_{\mathrm{S}}$ & $Z_{\mathrm{Ar}}$ & $Z_{\mathrm{Ca}}$ & $Z_{\mathrm{Fe}}$ & $Z_{\mathrm{Ni}}$ & $\chi^{2} /$ dof \\
\hline all field & MEKAL & $5.5_{-0.7}^{+0.9}$ & $6.6_{-0.3}^{+0.3}$ & $0.38_{-0.06}^{+0.06}$ & - & - & - & - & - & - & $205.1 / 178$ \\
\hline all field & VMEKAL & $5.5_{-0.8}^{+1.0}$ & $6.7_{-0.3}^{+0.4}$ & & $0.6_{-0.3}^{+0.3 *}$ & $0.2_{-0.2}^{+0.3 *}$ & $0.3_{-0.3}^{+0.6 *}$ & $0.6_{-0.6}^{+0.6 *}$ & $0.30_{-0.05}^{+0.05}$ & $0.1_{-0.1}^{+0.4 *}$ & $202.7 / 173$ \\
\hline Centre & MEKAL & $9.5_{-2.7}^{+3.8}$ & $6.2_{-0.4}^{+0.5}$ & $0.48_{-0.09}^{+0.10}$ & & & & & & & $198.9 / 161$ \\
\hline Centre & VMEKAL & $8.7_{-2.5}^{+4.2}$ & $6.2_{-0.5}^{+0.6}$ & & $0.7_{-0.4}^{+0.4 *}$ & $0.6_{-0.4}^{+0.5 *}$ & $1.0_{-0.8}^{+0.9 *}$ & $2.4_{-0.8}^{+0.9 *}$ & $0.40_{-0.08}^{+0.09}$ & $0.4_{-0.4}^{+0.6 *}$ & $192.2 / 156$ \\
\hline 1st Ring & MEKAL & $5.1_{-0.9}^{+1.2}$ & $6.6_{-0.5}^{+0.6}$ & $0.34_{-0.09}^{+0.10}$ & - & - & - & - & - & - & $214.4 / 178$ \\
\hline 1st Ring & VMEKAL & $5.0_{-0.9}^{+1.2}$ & $6.7_{-0.6}^{+0.6}$ & & $0.6_{-0.4}^{+0.4 *}$ & $0.3^{\dagger}$ & $1.0_{-0.9}^{+0.9 *}$ & $0.3^{\dagger}$ & $0.28_{-0.08}^{+0.08}$ & $0.1_{-0.1}^{+0.6 *}$ & $212.72 / 175$ \\
\hline 2nd Ring & MEKAL & $3.6_{-1.0}^{+1.5}$ & $6.7_{-0.8}^{+0.9}$ & $0.20_{-0.13}^{+0.13}$ & - & - & - & - & - & - & $120.4 / 142$ \\
\hline 2nd Ring & VMEKAL & $3.5_{-1.0}^{+1.6}$ & $6.7_{-0.9}^{+1.1}$ & & $0.8_{-0.6}^{+0.6 *}$ & $0.5_{-0.5}^{+0.6 *}$ & $0.3^{\dagger}$ & $0.6_{-0.6}^{+1.3 *}$ & $0.17_{-0.11}^{+0.11}$ & $0.8_{-0.8}^{+0.9 *}$ & $118.90 / 138$ \\
\hline 3rd Ring & MEKAL & $5.5_{-2.4}^{+4.8}$ & $9.1_{-2.8}^{+6.9}$ & $0.6_{-0.6}^{+0.6}$ & - & - & - & - & - & - & $79.6 / 72$ \\
\hline S. Blob & MEKAL & $4.6_{-1.2}^{+1.9}$ & $6.9_{-0.8}^{+1.0}$ & $0.24_{-0.14}^{+0.15}$ & - & - & - & - & - & - & $127.7 / 108$ \\
\hline S. Blob & VMEKAL & $4.5_{-1.2}^{+1.9}$ & $6.8_{-0.9}^{+1.2}$ & & $0.1_{-0.1}^{+0.6 *}$ & $0.5_{-0.5}^{+0.7 *}$ & $0.3^{\dagger}$ & $1.7_{-1.3}^{+1.6 *}$ & $0.20_{-0.11}^{+0.12}$ & $0.6_{-0.6}^{+1.0 *}$ & $126.03 / 104$ \\
\hline VSSRS & MEKAL & $3.3_{-1.4}^{+2.8}$ & $6.6_{-1.3}^{+1.8}$ & $0.31_{-0.25}^{+0.27}$ & - & - & - & - & - & - & $104.8 / 108$ \\
\hline N. VSSRS & MEKAL & $2.6_{-1.3}^{+2.9}$ & $7.1_{-1.4}^{+2.1}$ & $0.33_{-0.27}^{+0.28}$ & - & - & - & - & - & - & $117.8 / 108$ \\
\hline
\end{tabular}

* $1 \sigma$ error.

$\dagger$ Value fixed.

fitted the whole cluster. In a previous study, Pislar et al. (1997) observed a negative correlation between the X-ray gas temperature and $N_{\mathrm{H}}$. Here, we observe a much weaker correlation (Fig. 2), so the effect - temperature decrease towards the centre - is probably real and not an artefact of the fit. The metallicity is significantly higher in the central region than in the whole field. The ROSAT data gave the same results (Pislar et al. 1997). We detect the presence of $\mathrm{Si}, \mathrm{S}, \mathrm{Ar}, \mathrm{Ca}$ and Fe when we fit the data with a VMEKAL model. These results can be interpreted as due to the accumulation of neutral hydrogen in the vicinity of the cD galaxy, due to the presence of a cooling flow with star formation.

However, a single temperature plasma model is unable to provide a good fit for the spectrum of the central region of Abell 85 when the photon energy is less than $0.4 \mathrm{keV}$ : as is shown in Fig. 3a, there is a clear excess of soft X-ray photons.

Indeed, previous studies of Abell 85 have suggested that this cluster houses a strong central cooling flow (e.g. Stewart et al. 1984; Edge et al. 1992; Prestwich et al. 1995; Pislar et al. 1997; Lima Neto et al. 1997). Estimated values range from about 50 to $200 M_{\odot} /$ yr inside a cooling radius of $\sim 100-200 h_{50}^{-1} \mathrm{kpc}$. We can thus interpret the soft X-ray excess component as evidence for the presence of cool gas. Therefore we have fitted the central region spectrum with a 2-temperature gas model.

When we fix 2 or 3 parameters of the first thermal component, using the "all field" mean values, the fits are not significantly improved (cf. Table 3). However when we allow all the parameters to vary freely, the fit is significantly improved (the reduced $\chi^{2}$ is 1.13 instead of 1.23 ). Figure $3 \mathrm{~b}$ shows the best 2-temperature gas model fit. The parameters of the first component are roughly the same as that obtained in a single component fit, but the temperature of the second component is found to be very small (less than $0.1 \mathrm{keV}$ at $3 \sigma$ level). Thus, we recover the previous results based on the PSPC data reported in Lima Neto et al. (1997), in particular their 2-temperature fit of the central region, with the cooler component at $T=0.10 \pm 0.03 \mathrm{keV}$ (see Table 2 in their paper).

Since the central cD galaxy is also a radio source (as can be seen in Fig. 1), we tried a fit with a thermal component and a power law, in order to model an eventual non-thermal X-ray source (e.g. an Inverse Compton scattering of relativistic electrons with the microwave background photons). However, the inclusion of a power-law component does not improve the fit and we cannot detect such an emission.

\subsection{South Blob}

The South Blob region is a circle of 3.2 arcmin centred at the coordinates indicated in Table 1 . We fitted this region with both MEKAL and VMEKAL single-temperature models (Table 2) and a MEKAL plasma superposed to a power-law emission (Table 3 ). The latter was motivated by the presence of an extended radio emission seen on the 

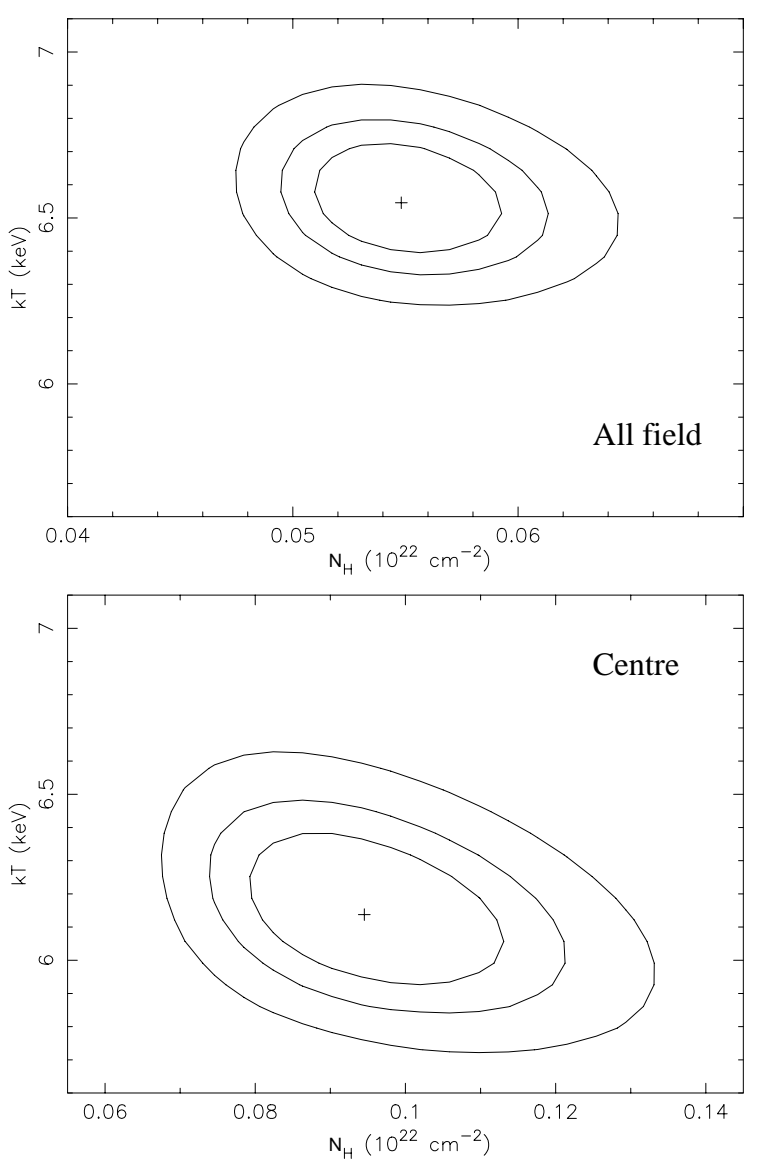

Fig. 2. Contours of chi-square values for the $N_{\mathrm{H}}-k T$ plane. These contours correspond to the best fits with the MEKAL model (cf. Table 2.) for "all field" (upper panel) and the centre of the cluster (lower panel). Notice that the $n_{\mathrm{H}}$ scale is different for the two plots. The contours shown are at the $1 \sigma, 2 \sigma$, and $3 \sigma$ confidence level

$90 \mathrm{~cm}$ VLA map (Fig. 1) and on the $326.5 \mathrm{MHz}$ OSRT map (Fig. 1 in Bagchi et al. 1998). Such an extended radio emission reveals the presence of relativistic electrons that will interact with the microwave background photons and produce an Inverse Compton X-ray emission, depending on the (a priori unknown) Lorentz factor and energy distribution of these electrons.

With both single-temperature models, the hydrogen column density is higher than in previous fits (Pislar et al. 1997) and marginally compatible with the Galactic value (3.08 $10^{20} \mathrm{~cm}^{-2}$, Dickey \& Lockman 1990). The hydrogen column density is even higher, however, when we use a MEKAL plus power-law model (although still compatible within their error bars). The temperature is higher than in the centre, being actually above the cluster mean temperature. With the MEKAL plus power-law model, the obtained temperature is even higher (but with larger error bars). Notice that, comparing the reduced $\chi^{2}$, an addition of a power-law component does not improve the fit.

The metallicity obtained in the South Blob is significantly lower than the mean value obtained for the whole cluster and is about half the value found in the centre of the cluster. This lower metallicity is also obtained with the iron abundance in the VMEKAL model. Besides iron, only $\mathrm{Ca}$ was detected with an abundance $1 \sigma$ above zero (but with a very large upper limit).

\subsection{VSSRS}

This is the region where a diffuse, very steep spectrum radio source (MRC 0038-096; see Bagchi et al. 1998) is observed.

Here, fitting a single plasma component, we obtain the same temperature and metallicity as in the whole field (cf. Table 2), even though the error bars are significantly larger due to the smaller count rate. The hydrogen column density is the same as the Galactic value.

In both the HRI and PSPC images it is possible to notice an X-ray excess in this region, spatially correlated with the diffuse VSSRS (Lima Neto et al. 1997). This Xray excess, interpreted as $\mathrm{IC} / 3 \mathrm{~K}$ emission, was estimated by Bagchi et al. (1998) by subtracting the thermal contribution from the total X-ray emission. The latter was computed using a detailed plasma model for Abell 85 (see Pislar et al. 1997).

Ideally, one would like to distinguish directly and unambiguously the IC/3K from the thermal X-ray emission. In principle, this could be done by fitting a composite model to the X-ray spectrum. Thus, we have fitted the X-ray spectrum in the VSSRS region with the sum of a MEKAL plus a power-law model, the latter representing the $\mathrm{IC} / 3 \mathrm{~K}$ emission. The results are summarized in Table 3. We obtained, for the $\mathrm{IC} / 3 \mathrm{~K}$ emission, a spectral index of 0.4 but the reduced $\chi^{2}$ for the composite model is not statistically better than that obtained with a single MEKAL model. In Fig. 4 we show the best fit model for the VSSRS. Extrapolating the model to higher energies, the IC/3K mechanism starts to dominate the total X-ray flux beyond $\sim 15 \mathrm{keV}$ (a well-known result), outside the range of the MECS detector.

The difficulty in distinguishing between thermal and non-thermal X-ray emission is due to two main factors: (i) the $\mathrm{IC} / 3 \mathrm{~K}$ emission is relatively weak compared to the thermal one and (ii) the bremsstrahlung emission is well approximated by a power-law in the energy interval observed by BeppoSAX.

We also tried a fit with a fixed value for the spectral index equal to 1, i.e., the same spectral index of the VSSRS between 10 and $100 \mathrm{MHz}$ (see Fig. 3 of Bagchi et al. 1998). Again, the fit is not significantly improved but the flux of the $\mathrm{IC} / 3 \mathrm{~K}$ is not negligible. Notice, however, that the best fit spectral index of 0.4 is consistent with the radio spectrum in the interval [10-40] MHz, where the spectrum seems to flatten.

Furthermore, we have modelled the IC/3K emission as a broken power-law, characterized by two power-law slopes and a breaking point. We could not obtain a robust fit letting the two slopes vary as free parameters. Therefore, 

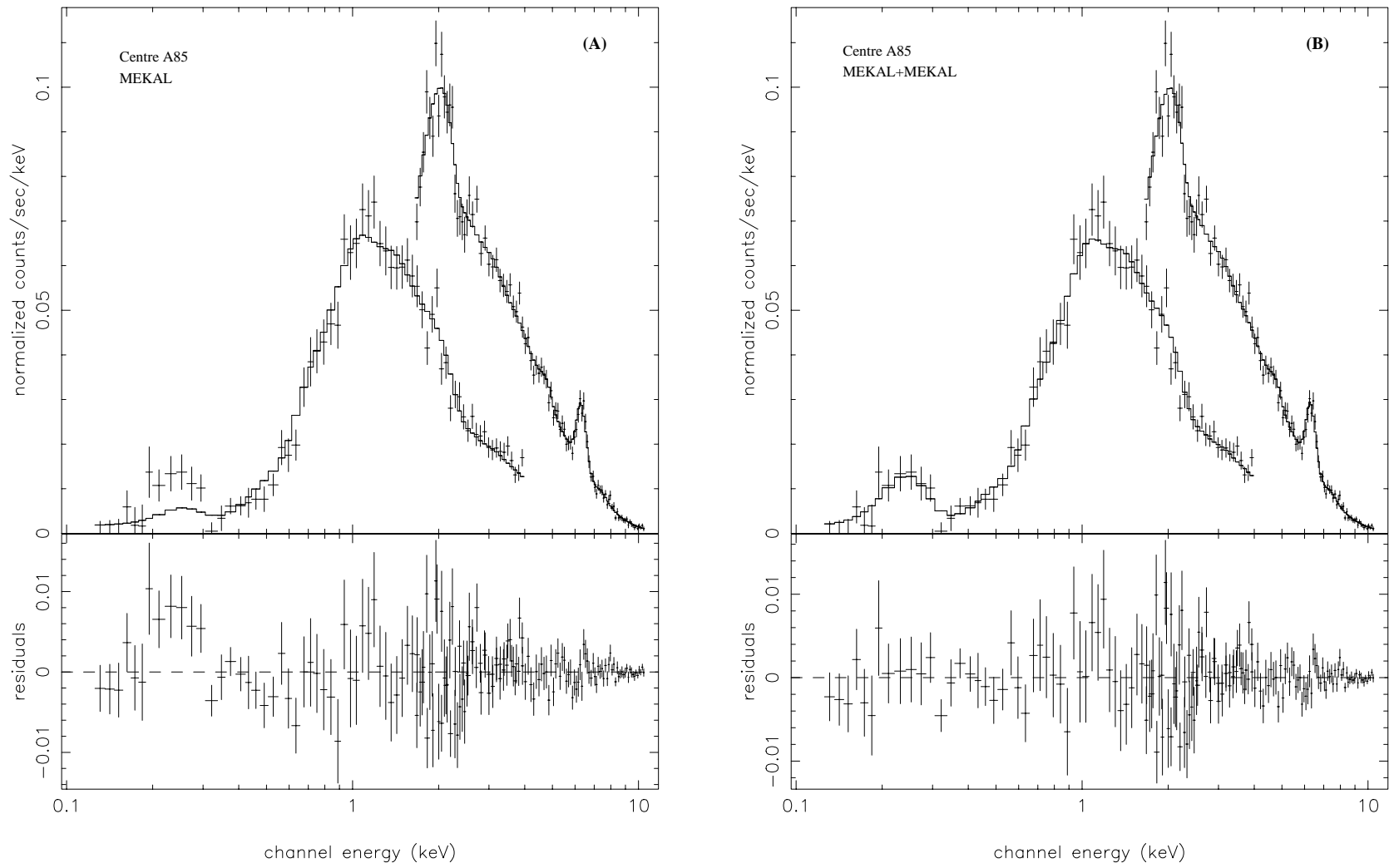

Fig. 3. Plasma model fits to the centre region of Abell 85. Left: single temperature MEKAL model. Right: two-temperature model. Notice the low energy photon excess in panel a) compared to the two-temperature model b)

Table 3. Two-component gas model results. The flux is without absorption, in units of $10^{-11} \mathrm{erg} \mathrm{s}^{-1} \mathrm{~cm}^{-2}$, where Flux 1 and Flux 2 refer either to the thermal and non-thermal components, respectively, or to two thermal components (in the MEKAL+MEKAL case). "2POW" means broken power-law. Errors are $3 \sigma$ except when explicitly stated

\begin{tabular}{|c|c|c|c|c|c|c|c|c|c|c|}
\hline Region & Models & $\begin{array}{c}N_{\mathrm{H}} \\
\left(10^{20} \mathrm{~cm}^{-2}\right)\end{array}$ & $\begin{array}{c}T_{1} \\
(\mathrm{keV})\end{array}$ & $\begin{array}{c}Z_{1} \\
\left(Z_{\odot}\right) \\
\end{array}$ & $\begin{array}{c}T_{2} \\
(\mathrm{keV}) \\
\end{array}$ & $\begin{array}{c}Z_{2} \\
\left(Z_{\odot}\right) \\
\end{array}$ & $\alpha$ & $\begin{array}{c}\text { Flux }_{1} \\
{[2-10] \mathrm{keV}}\end{array}$ & $\begin{array}{c}\text { Flux }_{2} \\
{[2-10] \mathrm{keV}}\end{array}$ & $\chi^{2} /$ dof \\
\hline Centre & MEKAL+MEKAL & $5.5^{\dagger}$ & $6.6^{\dagger}$ & $0.38^{\dagger}$ & $5.9_{-2.0}^{+3.7}$ & $1.0^{\dagger}$ & - & $3.55 \pm 0.25$ & $0.86 \pm 0.15$ & $228.41 / 162$ \\
\hline Centre & MEKAL+MEKAL & $9.5_{-2.7}^{+3.8}$ & $6.6^{\dagger}$ & $0.38^{\dagger}$ & $6.1_{-3.8}^{+5.5}$ & $>0.4$ & - & $0.52 \pm 0.04$ & $3.89 \pm 1.00$ & $198.98 / 160$ \\
\hline Centre & MEKAL + MEKAL & $11.8_{-2.9}^{+3.4}$ & $6.0_{-0.4}^{+0.4}$ & $0.48_{-0.09}^{+0.10}$ & $2.2_{-1.3}^{+6.7} 10^{-2}$ & $>0.0$ & - & $4.42 \pm 0.14$ & $<0.01$ & $178.70 / 158$ \\
\hline Centre & MEKAL+POW & $9.4_{-2.6}^{+4.5}$ & $6.2_{-0.4}^{+0.5}$ & $0.48_{-0.09}^{+0.10}$ & - & - & $1.0^{\dagger}$ & $4.42 \pm 0.14$ & $<0.01$ & $198.93 / 160$ \\
\hline S. Blob & MEKAL+POW & $5.3_{-1.9}^{+8.1}$ & $7.1_{-3.1}^{+1.7}$ & $0.25_{-0.16}^{+0.20}$ & - & - & $1.3_{-1.3}^{+1.7 *}$ & $1.47 \pm 0.24$ & $0.064 \pm 0.057^{*}$ & $127.05 / 106$ \\
\hline VSSRS & MEKAL+POW & $3.8_{-1.8}^{+5.2}$ & $6.7_{-1.4}^{+2.8}$ & $0.33_{-0.27}^{+0.59}$ & - & - & $1.0^{\dagger}$ & $0.52 \pm 0.09$ & $0.033^{\dagger}$ & $104.37 / 107$ \\
\hline \multirow[t]{2}{*}{ VSSRS } & MEKAL+POW & $3.5_{-1.5}^{+5.7}$ & $5.5_{-2.6}^{+4.3}$ & $0.35_{-0.29}^{+1.59}$ & - & - & $0.4_{-0.4}^{+4.8}$ & $0.44 \pm 0.14$ & $0.12 \pm 0.12$ & $104.04 / 106$ \\
\hline & & $\begin{array}{c}N_{H} \\
\left(10^{20} \mathrm{~cm}^{-2}\right)\end{array}$ & $\begin{array}{c}T_{1} \\
(\mathrm{keV})\end{array}$ & $\begin{array}{c}Z_{1} \\
\left(Z_{\odot}\right)\end{array}$ & $\alpha_{1}$ & $\begin{array}{l}\text { Break } \\
(\mathrm{keV}) \\
\end{array}$ & $\alpha_{2}$ & $\begin{array}{c}\text { Flux }_{1} \\
{[2-10] \mathrm{keV}}\end{array}$ & $\begin{array}{c}\text { Flux }_{2} \\
{[2-10] \mathrm{keV}}\end{array}$ & $\chi^{2} /$ dof \\
\hline VSSRS & MEKAL+2POW & $4.0_{-1.5}^{+1.9}$ & $7.3_{-1.4}^{+2.7}$ & $0.34_{-0.17}^{+0.23}$ & $1.0^{\dagger}$ & $>0$ & $1.85^{\dagger}$ & $0.52 \pm 0.09$ & $0.03 \pm 0.03$ & $103.71 / 106$ \\
\hline VSSRS & MEKAL +2 POW & $3.7_{-1.0}^{+1.5}$ & $3.9_{-1.4}^{+5.7}$ & $0.44_{-0.25}^{+0.43}$ & $0.4^{\dagger}$ & $7.4_{-0.7}^{+0.9 *}$ & $1.85^{\dagger}$ & $0.27 \pm 0.18$ & $0.28 \pm 0.01^{*}$ & $101.22 / 106$ \\
\hline
\end{tabular}

* $1 \sigma$ error.

$\dagger$ Value fixed.

we fixed the steeper slope, $\alpha_{2}$, equal to the observed slope of the radio spectrum in the range $[100-400] \mathrm{MHz}$, i.e., $\alpha_{2}=1.85$ (Bagchi et al. 1998) and fixed the shallower slope $\left(\alpha_{1}\right)$ either to 1.0 (from the radio spectrum) or to 0.4 , our best fit with a single power-law (cf. Table 3 ).
In the first case, with $\alpha_{1}=1$, the fit is only slightly better than that with a single power-law, and the derived $\mathrm{IC} / 3 \mathrm{~K}$ flux is much smaller than the thermal flux. Unfortunately, we cannot obtain a meaningful value for the breaking point. In the second case, $\alpha_{1}=0.4$, the fit is 


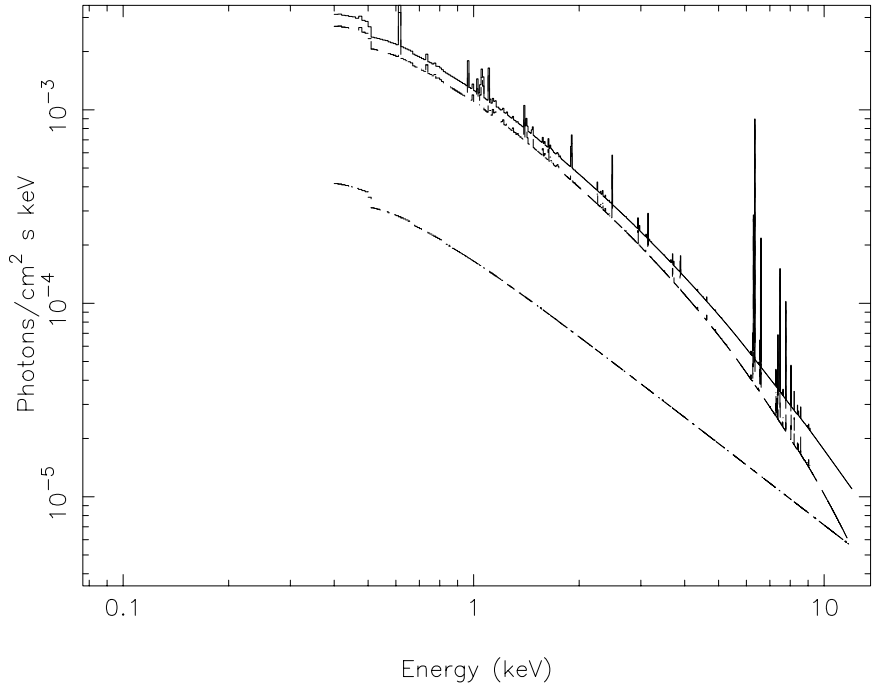

Fig. 4. Plasma model with two components, MEKAL and power-law, for the VSSRS region. The lower dash-dotted curve represents an Inverse Compton emission with spectral index $\alpha=0.4$; the dashed curve is a MEKAL model with temperature $5.5 \mathrm{keV}$ and abundance $0.35 Z_{\odot}$ (cf. Table 3 ). The continuous line is the sum of the components

still better and the breaking point is well determined at $7.4 \mathrm{keV}$. However, the derived fluxes for the thermal and the broken power-law components are almost the same, indicating the confusion in separating the thermal from the non-thermal emission.

Sarazin (1999) has computed the Inverse Compton spectra for a number of models with different relativistic electron populations. For the models with steady particle injection, the IC emission spectra in the combined MECS and LECS range would be well represented by a single power-law with slope $\alpha \approx(p / 2)$, where $p$ is the logarithm slope of the relativistic electron distribution (cf. Fig. 12 of Sarazin 1999).

For the models with no injection of electrons but with an initial population, the IC emission could only be detectable if the initial population were injected in the cluster at a redshift lower than 0.1 (cf. Fig. 13 of Sarazin 1999). In this case, a broken power-law is needed to reproduce the IC emission with $\alpha_{1} \approx(p-1) / 2$ and $\alpha_{2} \approx p$ (actually, the emission has an exponential cut-off and these slopes are only valid in the range $[0.1-10.0] \mathrm{keV})$.

\subsection{Other regions}

We made fits in the regions we called 1st, 2nd, 3rd Rings and N. VSSRS. In the 3rd ring and N. VSSRS regions, the error bars are quite large, due to the small number of counts and it is difficult to deduce strong results.

The hydrogen column density and metallicity decrease from the centre to the 2 nd ring, while the temperatures of the 1st and 2nd rings are larger than in the centre.

\section{Discussion}

\subsection{Estimation of magnetic fields}

In Sect. 3, we have attempted to separate the thermal and non-thermal X-ray emission components in the VSSRS and South Blob regions, where radio observations have detected diffuse synchrotron radiation, showing the presence of magnetic fields and relativistic electrons. Here, we estimate the volume averaged $B$ field in these zones, using the non-thermal fluxes (cf. Table 3). We wish to inject a cautionary note at this stage: in this section, the $B$ values are expressed as actual estimates, where we have used the nominal results given in Table 3 . However, given the non-thermal X-ray flux errors bars, these fluxes should be regarded as upper limits. Therefore, all our estimates of the $B$ field intensities should be ideally treated only as lower limits.

\subsubsection{The VSSRS region}

In the first case (MEKAL+POW), when the spectral indices of non-thermal X-ray and radio photons are both fixed at $\alpha_{1}=1$, the $\mathrm{IC} / 3 \mathrm{~K}$ flux $\left(f_{\mathrm{IC}}\right)$ is estimated to be $3.310^{-13} \mathrm{erg} \mathrm{s}^{-1} \mathrm{~cm}^{-2}$ in $2-10 \mathrm{keV}$ range and the radio flux $\left(f_{\mathrm{S}}\right)$ to be $6.610^{-14} \mathrm{erg} \mathrm{s}^{-1} \mathrm{~cm}^{-2}$ in $10-100 \mathrm{MHz}$ range. This leads to a field value of $B=1.34 \pm 0.24 \mu \mathrm{G}$.

In the second case, when we have allowed the spectral index to vary, we obtained the value of $\alpha_{1}=0.4$, with large error bars (Table 3 ). This value of the spectral index seems roughly consistent with a radio spectral shape in the range 10-45 MHz (Bagchi et al. 1998). In this case, $f_{\mathrm{IC}}=$ $1.210^{-12} \mathrm{erg} \mathrm{s}^{-1} \mathrm{~cm}^{-2}$ and $f_{\mathrm{S}}=4.410^{-14} \mathrm{erg} \mathrm{s}^{-1} \mathrm{~cm}^{-2}$. From these, we then obtain $B=0.40 \pm 0.13 \mu \mathrm{G}$.

Finally, we consider the more complex model where the non-thermal spectrum is represented by two power-law forms with a "spectral break" in-between $(\mathrm{MEKAL}+2 \mathrm{POW})$. When the low and the high frequency spectral segments are fixed a priori, with spectral indices $\alpha_{1}=1$ and $\alpha_{2}=1.85$, we once more obtain the magnetic field as $B=1.34 \pm 0.24 \mu \mathrm{G}$ (but note that the "break" value for $\mathrm{X}$-ray photons is not obtained).

However, when $\alpha_{1}$ is fixed at 0.4 and $\alpha_{2}$ is fixed at 1.85 , we obtain a well-constrained value $(7.4 \mathrm{keV})$ for the "break" in the IC $/ 3 \mathrm{~K}$ spectrum (Table 3 ). It is possible to relate this break to a similar break in the radio spectrum since both come from a common electron energy spectrum. The frequencies of $\mathrm{IC} / 3 \mathrm{~K}$ photons $\left(\nu_{\mathrm{IC}}\right)$ and of synchrotron photons $\left(\nu_{\mathrm{S}}\right)$ are related by:

$\nu_{\mathrm{IC}}(\mathrm{Hz})=4.8910^{10}(1+z) \nu_{\mathrm{S}}(\mathrm{Hz})[B(\mu \mathrm{G})]^{-1}$.

The decametric radio spectrum for the VSSRS shown in Fig. 3 of Bagchi et al. (1998) does show a sudden flattening or "break" in the spectrum at $\approx 30-40 \mathrm{MHz}$. Assuming $\nu_{\mathrm{IC}}=1.7710^{18} \mathrm{~Hz}($ or $7.4 \mathrm{keV})$ and $\nu_{\mathrm{S}}=(35 \pm 10) 10^{6} \mathrm{~Hz}$, we have another magnetic field estimate for the VSSRS from the last equation. This is obtained as $B=1.01 \pm$ $0.31 \mu \mathrm{G}$. 
Putting together the above, and using mean values and standard deviations derived above, results in $B \geq 0.9 \mu \mathrm{G}$. As per the caveat mentioned above, this really is the lower limit to the magnetic field value and should be treated as such. Our new estimate using the BeppoSAX data is consistent with our earlier estimate $(B=1.0 \pm 0.1 \mu \mathrm{G})$ using ROSAT data (Bagchi et al. 1998).

\subsubsection{The "South Blob" region}

Currently, due to insufficient radio data, the spectral index value for the emission from the South Blob is not available. However, the radio data from the Ooty Synthesis Telescope at $327 \mathrm{MHz}$ detects the total $450 \mathrm{mJy}$ diffuse radio flux from this zone (Bagchi et al. 1998). In Sect. 2, by model fitting, we have obtained an estimate of $6.410^{-13} \mathrm{erg} \mathrm{s}^{-1} \mathrm{~cm}^{-2}$ for the IC/3K flux and $\alpha=1.3$ for the spectral index. Assuming this value for the spectral index over the entire radio range $(10 \mathrm{MHz}$ to $10 \mathrm{GHz})$, we can then easily calculate the lower limit value for the magnetic field in this region, which is $B \geq 0.4 \mu \mathrm{G}$.

\subsection{Cluster merging at the South Blob}

The temperature detected in the South Blob is higher than in the other regions of Abell 85. This result is similar to that found by Markevitch et al. (1998) (cf. their Fig. 2) using ASCA data. They interpret the higher temperature in the region of the South Blob with the model proposed by Durret et al. (1998), that is, of a substructure falling in the main body of Abell 85. The higher temperature in this region, compared with either a region symmetrically opposed towards the north of Abell 85 or a region farther to the south, may be explained by a shock that heats the ICM. Although, with BeppoSAX, we cannot measure temperatures south of the South Blob or much north of the centre, the higher temperature that we do detect at the South Blob and the 2nd Ring tends to confirm this picture of a merging substructure.

We have also detected, with a $3 \sigma$ level, an excess of neutral hydrogen compared to the galactic value (from Dickey \& Lockman 1990). This excess, about 1.5 times the galactic value, also supports the scenario where gas is pouring in this region. Notice that the $N_{\mathrm{H}}$ measured over the entire 2nd Ring (which contains most of the South Blob) is substantially closer to the galactic value, suggesting that the localised excess at the South Blob region is real.

Interestingly, the South Blob presents a significantly lower abundance of metals compared to the other regions of Abell 85 and to its mean value. The 2 nd ring, which contains the South Blob, has also the same low metal abundance. If we admit the scenario of Durret et al. (1998), where a filament of very low surface brightness is falling into Abell 85, along the axis coming from Abell 87 and if Abell 87 is in fact composed of poor sub-clusters, then one can suppose that the gas in the filament falling into
Abell 85 has a rather low metallicity. This could explain the low abundance determined at the South Blob as the mixture of the $Z \sim 0.4 Z_{\odot}$ ICM from Abell 85 with a lower metal rich gas falling along the filament.

If the South Blob is indeed a place where a substructure is merging with the main body of Abell 85, then we may have a strong shock in this region. Such a shock is bound to accelerate the ICM electrons to relativistic energies and also may amplify stochastically the pre-existing magnetic fields (Enßlin et al. 1998; Sarazin 1999). These would in turn produce X-ray emission by IC/3K scattering and the radio synchrotron radiation. Our best fit with a power-law emission superposed to a thermal MEKAL model has virtually the same reduced $\chi^{2}$ as the single thermal model fit. In this fit, the non-thermal flux is only about $5 \%$ of the total flux and the power-law slope is $\alpha=1.3_{-1.3}^{+1.7}(1 \sigma)$. This corresponds to the slope of the relativistic electron energy spectrum of $p=3.6$.

\subsection{Metallicity}

The overall abundance detected with BeppoSAX is very close to the one found by Pislar et al. (1997) and it is the usual value found in clusters with the same temperature of Abell 85. The total abundance determined with the MEKAL model is quite well constrained, mainly by the prominent $\mathrm{Fe}-\mathrm{K} \alpha$ complex at $\sim 6.8 \mathrm{keV}$. The mean value obtained for the whole cluster is $0.38 Z_{\odot}$ with a $3 \sigma$ error of 0.06 .

The individual iron abundance is also well constrained using the VMEKAL model, and we systematically obtain a lower value for the Fe abundance compared to the mean abundance of all metals. The mean value for the whole field is 0.30 with a $3 \sigma$ error of 0.05 . Notice that the Fe abundance and the mean metal abundance are only marginally compatible with the $3 \sigma$ error bar - these abundances are different at the $2 \sigma$ level.

The above result is consistent with the fact that the individual abundances of other metals (except perhaps for $\mathrm{Ni}$ ) obtained with the VMEKAL model are usually higher than the iron abundance (cf. Table 4). In other words, the abundance ratio of $\alpha$-elements ( $\mathrm{Si}, \mathrm{S}, \mathrm{Ar}$ and $\mathrm{Ca}$ ) to iron is greater than one (in solar units). However, the $\mathrm{Ni} / \mathrm{Fe}$ abundance ratio is systematically lower than the $\alpha$-elements/Fe. This result must be taken very cautiously, given the large error bars of the $\alpha$-elements, and is most significant for the fits of the whole field and the centre region.

The results presented in Table 4 are somewhat in disagreement with those found by Mushotzky et al. (1996) (see also Loewenstein \& Mushotzky 1996) using ASCASIS data for four rich clusters of galaxies (A496, A1060, A2199 and AWM7). Although we also find an overabundance of $\mathrm{Si}$, our abundance rates for $\mathrm{S}, \mathrm{Ar}$ and $\mathrm{Ca}$ relative to iron are higher than theirs. The relative abundance of Si to Fe found here in Abell 85 is in remarkable agreement with the results of Fukazawa et al. (1998), also based on 
Table 4. Abundances relative to iron derived from Table 2 for whole field, the centre region and the first two rings. The numbers in brackets give the observed $1 \sigma$ interval

\begin{tabular}{c|ccccc}
\cline { 2 - 6 } \multicolumn{1}{c}{} & $\mathrm{Si}$ & $\mathrm{S}$ & $\mathrm{Ar}$ & $\mathrm{Ca}$ & $\mathrm{Ni}$ \\
\hline all field & 2.0 & 0.7 & 1.0 & 2.0 & 0.3 \\
& {$[1.0-3.0]$} & {$[0.0-1.7]$} & {$[0.0-3.0]$} & {$[0.0-4.0]$} & {$[0.0-1.7]$} \\
1.8 & 1.5 & 2.5 & 6.0 & 1.0 \\
2nt Ring & {$[0.8-2.8]$} & {$[0.5-2.8]$} & {$[0.5-4.8]$} & {$[4.0-8.2]$} & {$[0.0-2.5]$} \\
2.1 & - & 3.6 & - & 0.4 \\
{$[0.7-3.6]$} & - & {$[0.4-6.8]$} & - & {$[0.0-2.5]$} \\
4.7 & 2.9 & - & 3.5 & 4.7 \\
& {$[1.1-8.2]$} & {$[0.0-6.5]$} & - & {$[0.0-11.1]$} & {$[0.0-10.0]$} \\
\hline
\end{tabular}

ASCA data but for 40 clusters. Our results, $\mathrm{Si} / \mathrm{Fe} \approx 2$ and $T=6.6 \mathrm{keV}$, fall squarely on the correlation between the $\mathrm{Si} / \mathrm{Fe}$ abundance and cluster temperature (cf. their Fig. 3).

Our results tend to support the burst model for elliptical galaxies, where a strong galactic wind develops early in the galaxy history (Martinelli et al. 1999). These authors argue that, for the burst model, there should be an overabundance of $\alpha$-elements compared to iron, whereas for the continuous model, the abundance ratio is smaller than one at $z \sim 0$. The bi-modal model proposed by Elbaz et al. (1995) also predicts a higher abundance of Si compared to Fe. Following their conclusion, our results suggest that type II SN have the main role in the enrichment of the ICM. Fukazawa et al. (1998) also suggest that, if the $\mathrm{Si} / \mathrm{Fe}$ relative abundance is indeed correlated with the cluster temperature, then the role of type II SN should be more important for hotter clusters.

Based on upper limits on the red spectral line of [Ca II], Donahue \& Voit (1993) suggest that Ca is likely depleted onto dust grains in cluster cooling flows. The higher abundance of calcium derived by us may be the result of dust evaporation in the hot ICM - the calcium we observe in $\mathrm{X}$-rays is completely ionized.

\subsection{Cooling-flow}

We find a lower temperature in the central region, when compared to the mean cluster temperature, similar to Markevitch et al. (1998). They claim that it is a sign of a strong central cooling-flow. However, the difference in temperature we find is only of about $0.5 \mathrm{keV}$ and, given our $3 \sigma$ error bars, our results are also compatible with an isothermal temperature radial profile. Nevertheless, at $1 \sigma$ level, the temperature is not isothermal, having a profile that decreases towards the centre. The outermost ring shows a substantial increase in temperature, but its error bars are very large (due to the small number of counts).

Supporting the existence of a central cooling-flow is the fact that we have a better fit using a 2-temperature plasma model, with one of the components being very cold. This cold component was detected only in the central 2 arcmin region, as observed in most clusters with cooling-flows.
However, this cold gas at $T \lesssim 0.1 \mathrm{keV}$ cannot be related to the Extreme Ultra-Violet (EUV) excess found in some clusters by the EUVE satellite (Lieu et al. 1996; Lieu et al. 1999).

A cooling-flow picture is also supported by the hydrogen column density that we detect in the centre, much higher that the mean cluster value and at least two times higher than the galactic value from Dickey \& Lockman (1990). Notice that there is some correlation between $N_{\mathrm{H}}$ and $T$ (as seen in Fig. 2) in the sense that lower temperatures are obtained with higher hydrogen column densities.

The last evidence for a cooling-flow is the large metallicity found in the centre, significantly higher (at the $3 \sigma$ level) than the metallicity found elsewhere in the cluster.

The central region also shows a strong radio emission, both at 330 and $1400 \mathrm{MHz}$, however we detected no nonthermal emission. The superposed radio and optical data presented in our earlier work (cf. Fig. 2, Bagchi et al. 1998) show that this radio emission comes from the region of the central giant elliptical and another elliptical region $\sim 1.5$ arcmin to north-west of it. Possibly both these are active radio galaxies pouring relativistic electrons into the ICM.

\section{Conclusions}

We have presented new $\mathrm{X}$-ray data in the range $[0.1-$ 10.0] keV obtained with BeppoSAX. Upon analysing the spectrum of a circular region centred at the position of the VSSRS, we have derived the following mean values: $T=6.6 \pm 0.3 \mathrm{keV}, Z=0.38 \pm 0.06 Z_{\odot}$ and $N_{\mathrm{H}}=$ $5.5_{-0.7}^{+0.9} 10^{20} \mathrm{~cm}^{-2}$. The temperature is in good agreement with the value determined by ASCA (Markevitch et al. 1998), while the metallicity is in agreement with the value determined by ROSAT PSPC (Pislar et al. 1997).

Our main results are summarized below:

- We have derived an abundance ratio between $\alpha$ elements/iron greater than 1 and the iron metallicity is systematically lower than the mean metallicity. The over abundance of $\alpha$-elements may be an indication of an early enrichment of the ICM by type II SN in elliptical galaxies;

- The central region is better fitted with two temperature components, the lower temperature being lower than $\sim 0.1 \mathrm{keV}$. This supports the existence of a multiphase central cooling-flow;

- Since we could not separate unambiguously the thermal from the non-thermal X-ray emission, we have estimated, in a number of cases with different fitting procedures, the non-thermal X-ray flux. From these values and using the radio spectrum data, we derive a lower limit intensity of the extended magnetic field, $B \gtrsim 0.9 \mu \mathrm{G}$;

- The South Blob region shows a significantly lower metallicity than the rest of the cluster. Its temperature is also higher than its neighbouring regions. Such 
results support the picture of an in-falling stream merging with the main body of Abell 85 in that region. The VLA radio data shows the presence of both an extended magnetic field and relativistic particles in this zone. These may originate in an energy transfer from a strong shock that formed in a supersonic merger of intergalactic matter.

Acknowledgements. We thank F. Durret and D. Gerbal for fruitful comments and discussion. We thank Fabrizio Fiori for his valuable help with the BeppoSAX data reduction. This research has made use of SAXDAS linearized and cleaned event files (Rev.1.1) produced at the BeppoSAX Science Data Center. GBLN acknowledges financial support from the USP/COFECUB cooperation.

\section{References}

Anders, E., \& Grevesse, N. 1989, Geochim. Cosmochim. Acta, 53,197

Bagchi, J., Enßlin, T. A., Stalin, C. S., Humeshkar, N. B., \& Singh, M. 2001, Nature, submitted

Bagchi, J., Pislar, V., \& Lima Neto, G. B. 1998, MNRAS, 296, L23

Baldwin, J. E., \& Scott, P. E. 1973, MNRAS, 165, 259

Balucinska-Church, M., \& McCammon, D. 1992, ApJ, 400, 699

Boella, G., Butler, R. C., Perola, G. C., et al. 1997a, A\&AS, 122, 299

Boella, G., Chiappetti, L., Conti, G., et al. 1997b, A\&AS, 122, 327

Churazov, E., Gilfanov, M., Forman, W., \& Jones, C. 1996, ApJ, 471, 673

Cusumano, G., \& Mineo, T. 1998, On-flight calibration of the MECS vignetting

Dickey, J. M., \& Lockman, F. J. 1990, ARA\&A, 28, 215

Donahue, M., \& Voit, G. M. 1993, ApJ, 414, L17

Durret, F., Forman, W., Gerbal, D., Jones, C., \& Vikhlinin, A. 1998, A\&A, 335, 41

Edge, A. C., Stewart, G. C., \& Fabian, A. C. 1992, MNRAS, 258,177

Elbaz, D., Arnaud, M., \& Vangioni-Flam, E. 1995, A\&A, 303, 345
Enßlin, T. A., Biermann, P. L., Klein, I., \& Kohle, S. 1998, A\&A, 332, 395

Feenberg, E., \& Primakoff, H. 1948, Phys. Rev., 73, 449

Feretti, L., \& Giovannini, G. 1996, IAU Symp. 175, Extragalactic Radio Sources, ed. R. Ekers, et al. (Kluwer: Dordrecht), 333

Fiore, F., Guaianazzi, M., \& Grandi, P. 1999, Cookbook for BeppoSAX NFI spectral analysis, version 1.2, www.sdc.asi.it/pub/sax/doc/software_docs/saxabc_v1. 2.ps.gz

Fukazawa, Y., Makishima, K., Tamura, T., et al. 1998, PASJ, 50,187

Joshi, M. N., Kapahi, V. K., \& Bagchi, J. 1986, Proc. of NRAO Workshop on Radio Continuum Processes in Clusters of Galaxies, ed. C. P. O'Dea, \& J. M. Uson, 73

Kaastra, J. S., \& Mewe, R. 1993, A\&AS, 97, 443

Kronberg, P. P. 1994, Rep. Prog. Phys., 57, 325

Liedahl, D. A., Osterheld, A. L., \& Goldstein, W. H. 1995, ApJ, 438, L115

Lieu, R., Mittaz, J. P. D., Bowyer, S., et al. 1996, Science, 274, 1335

Lieu, R., Bonamente, M., Mittaz, J. P. D., et al. 1999, ApJ, 527, L77

Lima Neto, G. B., Pislar, V., Durret, F., Gerbal, D., \& Slezak, E. 1997, A\&A, 327, 81

Loewenstein, M., \& Mushotzky, R. F. 1996, ApJ, 466, 695

Markevitch, M., Forman, W. R., Sarazin, C. L., \& Vikhlinin, A. 1998, ApJ, 503, 77

Martinelli, A., Matteucci, F., \& Colafrancesco, S. 2000, A\&A, 354,387

Miniati, F., Ryu, D., Kang, H., et al. 2000, ApJ, 542, 608

Mushotzky, R. F., Loewenstein, M., Arnaud, K. A., et al. 1996, ApJ, 466, 686

Parmar, A. N., Martin, D. D. E., Bavdaz, M., et al. 1997, A\&AS, 122, 309

Pislar, V., Durret, F., Gerbal, D., Lima Neto, G. B., \& Slezak, E. 1997, A\&A, 322, 53

Prestwich, A. H., Guimond, S. J., Luginbuhl, C. B., \& Joy, M. 1995, ApJ, 438, L71

Raymond, J., \& Smith, B. W. 1977, ApJS, 35, 489

Rephaeli, Y., \& Gruber, D. E. 1988, ApJ, 333, 133

Sarazin, C. L. 1999, ApJ, 520, 529

Steward, G. C., Fabian, A. C., Jones, C., \& Forman, W. 1984, ApJ, 285, 1 